\title{
Perinatal loss and psychological support
}

\section{Sani, L. \& Bacqué, M-F.}

Department of Clinical Psychology, University of Strasbourg, France

Email: Isani@unistra.fr

\section{Introduction}

Worldwide, the number of children who died during the third trimester of pregnancy is approximately 2.6 million.

In France the child mortality rate is of 3,9 in 2018 per 1,000 live births (1).

Child loss is an event that is still very frequent in our society and the psychological consequences can be serious and lasting. Parents can develop Complicated Grief disorder (CG), depression, anxiety and Post-Traumatic Stress Disorder (PTSD). In particular, in Western World women are at greater risk of psychiatric diseases and hospitalizations, even thirty-five years after the loss (2).

\section{Objectives}

Our study is a longitudinal research: 29 French bereaved parents have been met for 4 time in 12 months:

- T1, as first meeting:

- T2 after 3 months;

- T3 and T4 after 6 months and one year from the first time.

The goal is to evaluate the psychological consequences and the effectiveness of therapeutic support, that is individual and group therapy and participation on social networks' groups (considered by parents as therapeutic).

\section{Participants}

29 parents (8 men and
21 women) with an
average age of 31 years
old;
participants live in couple $(38,7 \%)$ with a professional diploma $(41,9 \%)$ and a master's degree $(22,5 \%)$;

- Only $19 \%$ of the participants had already become a parent, of at most other 2 children.

- 21 children are females $(67 \%)$ and 11 males $(34 \%)$;

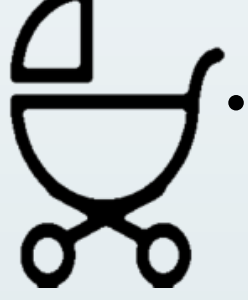
$44 \%$ of death occurred due to medical problems and $35 \%$ for a late termination of pregnancy;

- Only $22 \%$ of children lived (up to a maximum of 96 days);

- $78 \%$ died during pregnancy, starting from the twenty-second week of amenorrhea.

\section{Methodology}

The methodology is quantitative and qualitative.

We designed a descriptive study with:

- the Inventory of Complicated Grief (ICG), to assess the risk of complicated grief disorder;

- the General Health Questionnaire-28 (GHQ-28), a global self-assessment questionnaire for health disorders.

\section{Results}

The risk of developing a CG has decreased over time $(p<0.5)$. The mean of ICG is 28 , non reaching the reference cut-off after one year $(<25)$.

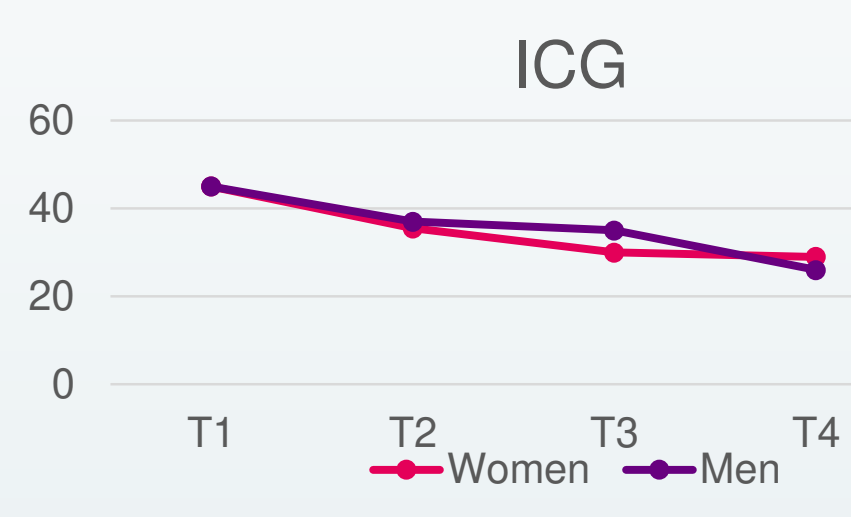

The data showed that for those who are at risk of developing a CG (ICG's mean=49), support groups are essential $(p<0.5)$, in particular at the beginning.

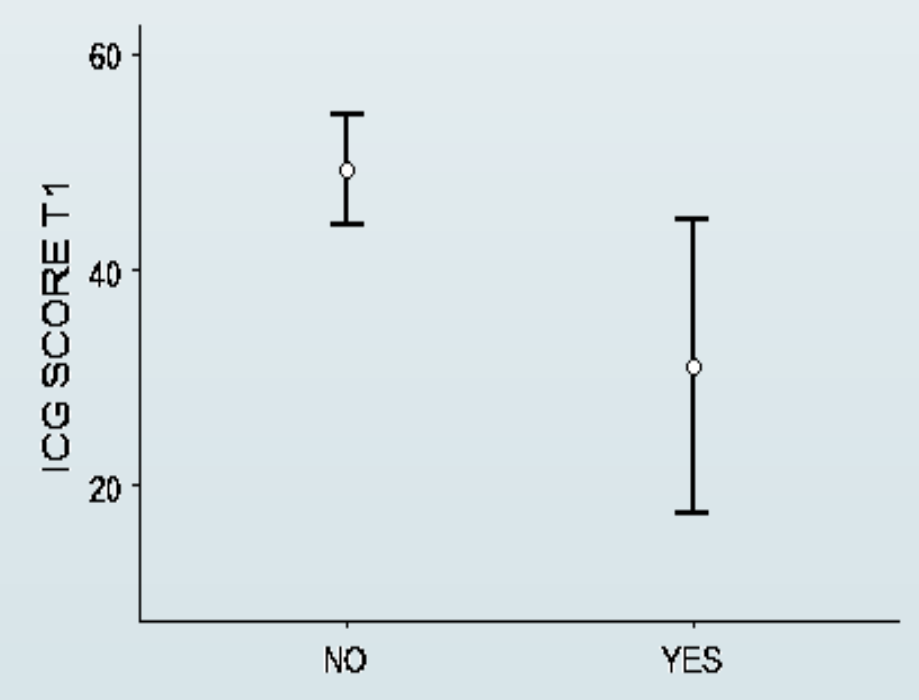

$(95 \% \mathrm{Cl})$

\section{Participation support group T1}

There were no significant differences compared to psychotherapy.

Instead, an active and prolonged participation on social networks may be a risk for a possible development of depressive disorder in women $(p<0.1)$ in $\mathrm{T} 4$.

\section{Conclusion}

CG is still an important risk for parents, without any difference between mothers and fathers.

The data show how the support group is an important protective factor for perinatal loss. Contrary to the expectations of the parents, who consider the use of internet as an alternative source of comfort and comparison, it does not replace therapeutic action or social support and recognition, which are fundamental for accepting the loss and completing the work of mourning.

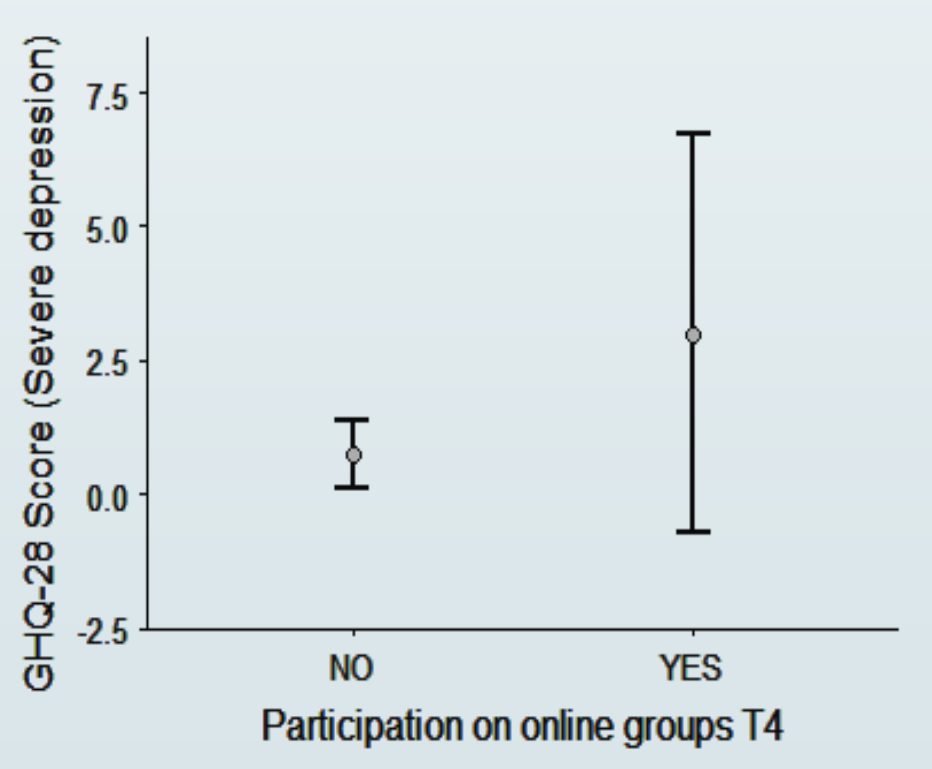

\section{References}

1. Insee. Taux de mortalité infantile en 2018 [Internet]. Insee - Institut national de la statistique et des études économiques. 2018 [cited 2019 Mar 26].

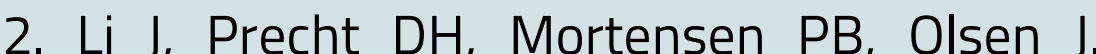
Mortality in parents after death of a child in Denmark: a nationwide follow-up study. The Lancet. 2003 Feb 1;361(9355):363-7. 